\title{
Development and Application of an ELISA Assay Using Excretion/Secretion Proteins from Epimastigote Forms of T. cruzi (ESEA Antigens) for the Diagnosis of Chagas Disease
}

\author{
Mariolga Berrizbeitia,, ${ }^{1,2}$ Milagros Figueroa, ${ }^{1}$ Brian J. Ward, ${ }^{3}$ Jessicca Rodríguez, \\ Alicia Jorquera, ${ }^{4}$ Maria A. Figuera, ${ }^{5}$ Leomerys Romero, ${ }^{4}$ and Momar $\mathrm{Ndao}^{3}$ \\ ${ }^{1}$ Laboratorio de Diagnóstico Serológico en Enfermedades Infecciosas, Postgrado en Biología Aplicada, Universidad de Oriente, \\ Núcleo de Sucre, Cumana 6101, Venezuela \\ ${ }^{2}$ Instituto de Biomedicina y Ciencias Aplicadas, Universidad de Oriente, Núcleo de Sucre, Cumana 6101, Venezuela \\ ${ }^{3}$ National Reference Centre for Parasitology, Research Institute of the McGill University Health Centre, Montreal General Hospital, \\ 1650 Cedar Avenue R3-137, Montreal, QC, Canada H3G 1A4 \\ ${ }^{4}$ Centro de Investigaciones en Ciencias de la Salud, de la Universidad de Oriente, Núcleo de Anzoátegui, Barcelona 6001, Venezuela \\ ${ }^{5}$ Departamento de Bioanálsis, Universidad de Oriente, Núcleo de Sucre, Cumana 6101, Venezuela
}

Correspondence should be addressed to Momar Ndao, momar.ndao@mcgill.ca

Received 5 May 2012; Accepted 14 August 2012

Academic Editor: Edecio Cunha-Neto

Copyright (c) 2012 Mariolga Berrizbeitia et al. This is an open access article distributed under the Creative Commons Attribution License, which permits unrestricted use, distribution, and reproduction in any medium, provided the original work is properly cited.

\begin{abstract}
An indirect enzyme-linked immunoabsorbent assay (ELISA) for Trypanosoma cruzi was developed using epimastigote secretion/excretion proteins (ESEA antigens) obtained from axenic culture supernatants. A panel of 120 serum samples from subjects with confirmed Chagas disease $(n=50)$, healthy controls $(n=50)$, and patients with other parasitic diseases $(n=20)$ was

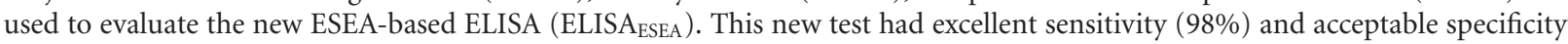
$(88 \%)$. Cross-reactivity was observed largely in sera from subjects with Leishmania and Ascaris infections. Using Western blotting and epimastigotes from two distinct T. cruzi isolates, several polypeptide bands with molecular masses ranging from 50 to $220 \mathrm{kDa}$ were detected in pooled chagasic sera. However, the band pattern for each isolate was different. These data suggest that an inexpensive and technically simple ELISA based on ESEA antigens is a promising new tool for the diagnosis of Chagas disease.
\end{abstract}

\section{Introduction}

Chagas disease, discovered by Dr. Carlos Chagas in 1909, is caused by the protozoan parasite, Trypanosoma cruzi. This parasite can be transmitted by triatomine vectors, blood transfusions, laboratory accidents, organ transplantation, or through vertical transmission. The disease is endemic in 18 Latin American countries. In 2008, the World Health Organization (WHO) estimates that $\sim 8$ million people were infected resulting in $\sim 11000$ deaths per year [1].

Despite decades of effort, there is still no gold standard test for the diagnosis of Chagas disease and several international organizations have recently emphasized the need for improved serodiagnostic tests [1]. To this end, many companies and research groups have developed a range of parasitologic, serologic, and nucleic acid-based assays. The most common methods include the indirect immunofluorescence assay (IFA), indirect haemagglutination assay (IHA), and the enzyme-linked immunosorbent assay (ELISA) [2]. The target antigens in these latter assays have included whole fixed parasites [3], 67 and $90-\mathrm{kDa}$ lectin purified proteins $[4,5]$, synthetic peptides [6], recombinant proteins [7-14], and trypomastigote excreted/secreted antigen (TESA). Of these antigens, TESA proteins have shown particular promise in different assay formats (ELISA-TESA, Western blot) and in several different laboratories and geographic settings [1519]. Unfortunately, the production of TESA antigen requires adequate cell culture facilities and other sophisticated 
infrastructure that are not always available to laboratories in resource-poor settings. Thus, in Latin American countries, diagnostic and research laboratories need practical, economical, and accurate tests that do not require special equipment [20]. Axenic growth of epimastigotes has long been used as a source of whole or purified T. cruzi antigens due to its simplicity and high antigen yields [15, 20-24]. To our knowledge, an ELISA based upon the excreted-secreted antigens of these epimastigote cultures has never been formally tested. The aim of the present study was to develop a simple, economical, and accurate ELISA using T. cruzi epimastigote secreted/excreted antigens (ELISA-ESEA) for the diagnosis of Chagas disease in areas with limited resources.

\section{Methods}

2.1. T. cruzi Strain. Epimastigote forms of T. cruzi MHOM/ $\mathrm{VE} / 08 / \mathrm{AU}$ isolate were obtained from an acute chagasic patient at the Tropical Medicine Center (Universidad de Oriente, Venezuela) and the RHO/VE/03/RG1 isolate was obtained from a vector (Rhodnius prolixus) captured in $\mathrm{La}$ Llanada de Cangua, Arismendi municipality, Sucre state, Venezuela. Both isolates were confirmed as T. cruzi by parasitological and molecular biology methods [25, 26]. The isolates were classified as TcI, widely distributed in Venezuela, and maintained on blood agar with monthly passages and adapted to axenic cultures.

2.2. Axenic Cultures T. cruzi. Epimastigotes of the T. cruzi (isolates $\mathrm{MHOM} / \mathrm{VE} / 08 / \mathrm{AU}$ and $\mathrm{RHO} / \mathrm{VE} / 03 / \mathrm{RG} 1$ ) were grown in Schneider's insect medium with L-glutamine (pH 6.9) (Sigma, St. Louis, MO, USA) supplemented with $0.6 \%$ calcium chloride and sterilized by filtration. After sterilization, heat-inactivated fetal bovine serum (FBS; Internegocios S.A. Buenos Aires, Argentina: final 10\%) and penicillin/streptomycin (Sigma, St. Louis, MO, USA: final $1 \%$ ) were added (complete media). Epimastigotes were collected during the logarithmic growth phase, as previously described [15].

2.3. ESEA Proteins. ESEA proteins from axenic cultures were harvested during logarithmic growth using a protocol adapted from the production of TESA antigens described by Berrizbeitia et al. [16]. Briefly, epimastigotes collected on the 7 th day of growth were washed four times with 3 volumes of Schneider's insect medium without FBS (complete media) and centrifuged $2,000 \mathrm{~g}$ for $10 \mathrm{~min}$ at $4^{\circ} \mathrm{C}$. The pellet was resuspended in $10 \mathrm{~mL}$ of complete media without FBS. After 4 days of incubation at $27^{\circ} \mathrm{C}$, the epimastigotes were centrifuged at 2,800 $\mathrm{g}$ for $20 \mathrm{~min}$ and the supernatant (ESEA proteins) was harvested and filtered through a Millipore membrane $(0.22 \mu \mathrm{m}$; Millipore, Bradford, MA, USA). Proteins were used immediately or stored at $-80^{\circ} \mathrm{C}$. Protein content of the ESEA was quantified using the Bradford assay (BioRad, Hercules, CA, USA).

2.4. SDS-PAGE. ESEA proteins were evaluated by SDSPAGE carried out on $0.8 \mathrm{~mm}$ thick slabs containing $10 \%$ polyacrylamide according to Laemmli [27]. Electrophoresis was performed for $2 \mathrm{~h}$ in Tris-glycine electrode buffer (25 mM Tris, $192 \mathrm{mM}$ glycine, $0.1 \%$ SDS, $\mathrm{pH}$ 8.3) at a constant voltage of $100 \mathrm{~V}$. Silver staining was employed for protein visualization. Molecular weight markers (BioRad, Hercules, CA, USA) were included in each run.

2.5. Western Blot Analyses. Proteins separated by SDS-PAGE were transferred to nitrocellulose sheets (MFS, Pleasanton, CA, USA) according to Towbin et al. [28] using a minitank electroblotter (BioRad, Hercules, CA, USA). The transfer was performed for $1 \mathrm{~h}$ at $4^{\circ} \mathrm{C}$ in $25 \mathrm{mM}$ Tris, $192 \mathrm{mM}$ glycine, and $20 \% \mathrm{v} / \mathrm{v}$ methanol $(\mathrm{pH} 8.3)$ at a constant current of $0.25 \mathrm{~A}$. Membranes were blocked overnight at $4^{\circ} \mathrm{C}$ with phosphate buffered saline (PBS) containing 5\% skimmed milk (Parmalat) and $0.1 \%$ Tween 20 (blocking solution) and then incubated for $2 \mathrm{~h}$ with a pool of confirmed positive chagasic sera diluted $1: 400$ in the blocking solution. Following four 5-min washes of PBS containing 0.05\% Tween 20 (PBST), the membranes were incubated for $2 \mathrm{~h}$ with the appropriate dilution of horseradish peroxidase (HRP)-conjugated goat antihuman IgG (Perkin Elmer Life Science, Boston, MA, USA) diluted in PBS containing 5\% skimmed milk and $0.1 \%$ Tween 20. The membranes were then washed four times with PBST, and the immune complexes were revealed using diaminobenzidine (Sigma, St. Louis, MO, USA).

2.6. Serum Samples. A panel of 120 serum samples was used in this study. Fifty were obtained from Venezuelan subjects with Chagas disease, as confirmed by a battery of three different serological tests, including immunofluorescence, indirect hemagglutination, and ELISA, at the National Chagas Immunodiagnosis Laboratory (NCIL, Maracay, Venezuela). Samples were considered positive if the results of two out of the three assays were positive. The other 70 serum samples (controls) included 50 that were negative for all three serological tests and 20 from individuals with the following parasitic diseases: leishmaniasis $(n=10)$, ascariasis $(n=8)$, strongyloidiasis $(n=1)$, and trichuriasis $(n=1)$, obtained from the NCIL (Maracay, Venezuela) and the Diagnostic Laboratory for Infectious Diseases (Universidad de Oriente, Núcleo de Sucre, Venezuela).

2.7. ESEA-Based ELISAs. The coating of 96 well-polystyrene plates (Immulon 2; Thermo Labsystems, Franklin, MA, USA) was accomplished by incubating ESEA from the AU isolate $(5,0 \mu \mathrm{g} / \mathrm{mL})$ at $4^{\circ} \mathrm{C}$ overnight $(100 \mu \mathrm{L} /$ well $)$ in $1 \mathrm{M}$ sodium carbonate buffer $(\mathrm{pH} 9.6)$. The plates were then washed four times with PBS ( $\mathrm{pH} 7.4 ; 0.01 \mathrm{M}$ phosphate buffer, $0.14 \mathrm{M} \mathrm{NaCl}$ ) containing $0.05 \%$ Tween 20 (A\&C, American Chemicals Ltd., St-Laurent, QC, Canada) (PBST) and blocked for $1 \mathrm{~h}$ at $37^{\circ} \mathrm{C}$ with PBS containing 5\% bovine serum albumin (Sigma, St. Louis, MO, USA) and $0.1 \%$ Tween 20. Sera were diluted 1:400 in the corresponding blocking solution $100 \mu \mathrm{L} /$ well and incubated for $1 \mathrm{~h}$ at $37^{\circ} \mathrm{C}$. These dilutions were selected to permit optimal differentiation between positive and negative control sera, as tested by checkerboard titration (data not shown). Assays 
were completed with an optimal dilution of horseradish peroxidase-conjugated goat anti-human immunoglobulin G (IgG; $100 \mu \mathrm{L} /$ well; Perkin-Elmer Life Science, Boston, $\mathrm{MA}$, USA) for $30 \mathrm{~min}$ at $37^{\circ} \mathrm{C}$, four washes with PBST, and a final incubation with 3,3',5,5'-tetramethylbenzidine ( $100 \mu \mathrm{L} /$ well; Serologicals Corporation, Billerica, MA, USA) for $10 \mathrm{~min}$ at room temperature. The reaction was stopped with $1 \mathrm{~N} \mathrm{H}_{2} \mathrm{SO}_{4}(50 \mu \mathrm{L} /$ well). Optical density (OD) was measured at $450 \mathrm{~nm}$ using an automated ELISA reader (Biotrak II, Amersham Biosciences, Cambridge, UK). All experiments were performed in duplicate on different days. Results were only accepted when the coefficient of variation within and among plates was $<15 \%$; otherwise, the samples were retested. The cut-off value for positivity was determined using a receiver-operating characteristic (ROC) curve analysis to establish the optimal sensitivity and specificity for the assay.

\section{Results}

3.1. ESEA Production. ESEA antigens were obtained from Schneider's insect medium supernatant on the fourth day of culture without purification procedures. The protein concentration in $60 \mathrm{~mL}$ of the axenic culture was $16.18 \mu \mathrm{g} / \mathrm{mL}$. The parasite density at the time of harvest was $1 \times$ $10^{7}$ parasites $/ \mathrm{mL}$.

3.2. Identification of ESEA Proteins in the Supernatants of Axenic Cultures. We performed SDS PAGE and WB analysis with pooled chagasic sera for the two isolates (MHOM/VE/08/AU and RHO/VE/03/RG1) to identify the immunoreactive polypeptide bands present in the supernatant of axenic T. cruzi cultures. The ESEA proteins, separated by SDS-PAGE and visualized with silver staining (Figure 1), varied both in number and molecular weights between the parasite isolates. The RG1 isolate had more protein bands than the $\mathrm{AU}$ isolate but there also appeared to be shared bands of approximately 25, 45, 66, and $200 \mathrm{kDa}$. Western blots also revealed the presence of immunogenic high molecular weight proteins, some of which were not visualized by silver stain of the SDS-PAGE. The pooled chagasic sera recognized ESEA polypeptides from both isolates, with molecular weights from 50 to over $200 \mathrm{kDa}$. Moreover, a pool of sera from healthy individuals did not react with the ESEA polypeptide bands (Figure 2). To our knowledge, this is the first paper that has both identified and showed the immunogenicity of ESEA proteins that could be used for the diagnosis of Chagas disease under different formats.

3.3. ELISA ESEA in Patient Samples. The ELISA ESEA was standardized using the MHOM/VE/08/AU isolate since this isolate had been obtained from an acute case of Chagas disease. Using checkerboard titrations, the best antigen concentration, as well as dilutions of the primary and secondary antibodies was determined to be $5 \mu \mathrm{g} / \mathrm{mL}, 1 / 400$, and $1 / 32000$, respectively. After assay optimization, the mean optical density (OD) values for the pooled chagasic sera

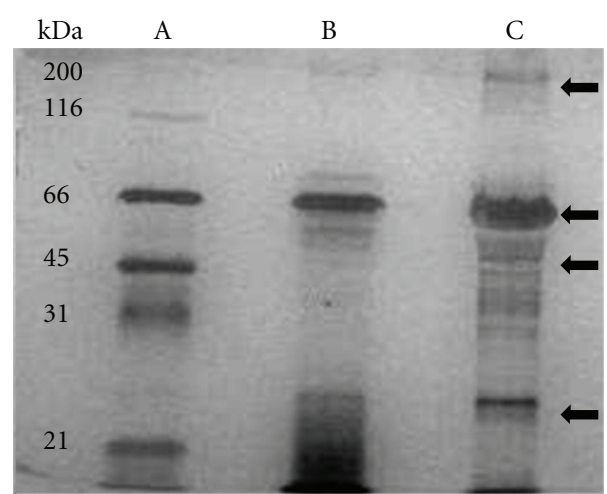

FIGURE 1: SDS-PAGE separation of the ESEA proteins visualized by silver staining. (A) (standard), (B) AU isolate, and (C) RG1 isolate. Arrows: common bands in both isolates.

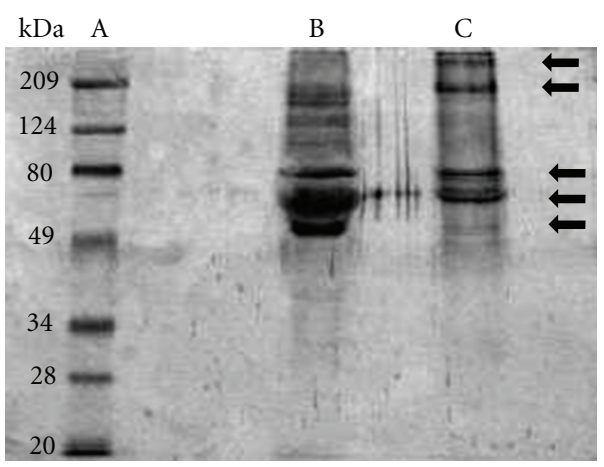

(a)

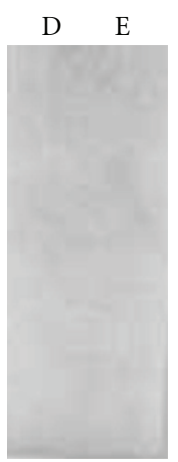

(b)
FIGURE 2: Western blot analysis of sodium dodecyl-polyacrylamide gel electrophoresis-separated ESEAs: Au and RG1 T. cruzi isolates. (A) standard, (B) RG1 isolate, and (C) AU isolate. The dot was probed with a pool of sera from patients with Chagas disease (B and C) and with a pool of control-negative sera RG1 and Au isolate, (D and $\mathrm{E}$ ), respectively.

and negative control sera were 1.753 and 0.212 , respectively (approximately $8: 1$ ). Generally, ELISA standardization requires this ratio to be at least $5: 1$ and a ratio of $>10$ is considered to be excellent [29].

Forty-nine out of the 50 serum samples from subjects with confirmed Chagas disease were reactive in the ELISA ESEA when an OD cut-off value of 0.600 was used (sensitivity, 98\%; 95\% confidence interval, 96 to $100 \%$ ) (Table 1). Six of the sera from healthy individuals (6/50) (12\%), four from patients with leishmaniasis (4/10) (40\%), three with ascariasis (3/8) (37.5\%) and 1 with strongyloidiasis [100\%] were reactive in this assay (specificity, 80\%). The mean OD values for chagasic sera, the negative control sera, Leishmania-positive sera and sera, from other parasitosis were $1.099 \pm 0.323$ (range, 0.474 to 1.888 ), $0.339 \pm 0.222$ (range, 0.073 to 1.129 ), $0.617 \pm 0.244$ (range, $0.370-1.137$ ), and $0.492 \pm 0.275$ (range, 0.250 to 0.921 ), respectively, (Table 2). Cut-off values between ODs of 0.40 to 0.60 yielded excellent sensitivity (98 to100\%) (Table 2). The cut-off value 0.6 was determined after using a receiver operating curve 
TABle 1: Sensitivity, specificity, positive predictive values, and negative predictive values of the ESEA-ELISA at various arbitrary OD cut-off values.

\begin{tabular}{lc}
\hline OD cut-off & ESEA-based assay \\
Values and parameters & $74(62-86)$ \\
\hline 0.400 & 100 \\
Specificity (\%) (95\% CI) & 100 \\
Sensitivity (\%) (95\% CI) & $79(69-89)$ \\
NPV (\%) (95\% CI) & \\
PPV (\%) (95\% CI) & $80(69-91)$ \\
\hline 0.500 & $98(94-100)$ \\
Specificity (\%) (95\% CI) & $98(93-100)$ \\
Sensitivity (\%) (95\% CI) & $83(73-93)$ \\
NPV (\%) (95\% CI) & \\
PPV (\%) (95\% CI) & $88(79-97)$ \\
\hline 0.600 & $98(96-100)$ \\
Specificity (\%) (95\% CI) & $98(94-100)$ \\
Sensitivity (\%) (95\% CI) & $89(81-97)$ \\
NPV (\%) (95\% CI) &
\end{tabular}

NPV: negative predictive value.

PPV: positive predictive value.

CI: confidence interval.

(ROC) (Figure 3). This gave the best values for sensitivity $(98 \%)$, specificity $(88 \%)$, positive predictive value (PPV, $89 \%$ ), and negative predictive value (NPV, 98\%) (Table 2).

\section{Discussion}

To our knowledge, we are the first to describe the possible use of ESEA antigens for the diagnosis of Chagas disease. ESEA antigens are simple and inexpensive to produce and protein yields can be very high. In addition, these antigens show an excellent sensitivity and an acceptable specificity in the ELISA format. In this study, we evaluated the use of ESEA antigens that could feasibly be produced in laboratories with scarce financial resources. For this reason, we adapted the protocol for the production of TESA antigens that have previously been shown to be excellent for the diagnosis of Chagas disease, in order to produce ESEA antigens [16]. Although TESA antigens represent an excellent diagnostic alternative for $T$. cruzi infections, they must be produced in cell cultures and thus require expensive laboratory equipment and infrastructure. This tends to increase the cost of producing TESA antigens, thus limiting their use in Latin America where resources are scarce. One of the most important advantages of ESEA antigens in relation to epimastigote-based ELISAs is that ESEA antigens are very stable and do not need protease inhibitors. Furthermore, reactivity is retained for approximately one year after production. Another advantage of ESEA antigens compared to other antigenic preparations from epimastigotes is the easy production since they donot need to be sonicated or follow other biochemical procedures to obtain usable antigenic preparations $[20,30,31]$.

The yield obtained for ESEA antigens was high enough to be usefully applied in diagnostic serological tests, giving an adequate quantity of ESEA antigens for diagnosis even with a low production of epimastigotes. Umezawa et al. [18] reported a similar concentration of excreted/secreted proteins in the supernatant of Vero cells $(30-40 \mu \mathrm{g} / \mathrm{mL})$ and demonstrated their usefulness for the diagnosis of Chagas disease.

In this study we demonstrated that ESEA antigens are a mix of polypeptide bands ranging from 20 to $220 \mathrm{kDa}$ that are secreted/excreted by epimastigote forms into the supernantant of axenic media. These ESEA antigens are immunogenic and the sera taken from the chagasic patients recognized these protein bands in a range from 50 to $220 \mathrm{kDa}$. To our knowledge, this is the first study that demonstrates the immunoreactivity of ESEA proteins. Moreover, using just two isolates, we were able to demonstrate that the bands vary in number and relative molecular weight. Kesper Jr. et al. [17] also demonstrated a unique banding pattern that is characteristic for each strain or isolate in T. cruzi trypomastigote exoantigens.

After demonstrating the detection of ESEA antigens by a pool of chagasic sera in the Western blot format, we evaluated the usefulness of these proteins for the diagnosis of Chagas disease in the ELISA format. The ESEA-ELISA using the AU strain was $98 \%$ sensitive and $88 \%$ specific, with the latter value being due to cross-reactions with sera from patients with leishmaniasis and other parasitosis. These results are similar to those of other investigations. Cannnova et al. [32] standardized an ELISA assay using a crude epimastigote antigen; the sensitivity and specificity of the test was $96 \%$ and 97\%, respectively. Moreover, Telles et al. [33] used T. cruzi ubiquitin as a differential diagnostic antigen to distinguish tripanosomiasis and leshmaniosis with an ELISA test at a sensitivity and specificity of $98 \%$ and $93.8 \%$, respectively, Additionally, Schechter et al. [34] developed an ELISA assay using a $90 \mathrm{kDa}$ purified protein, the sensibility and specificity of this assay are $96.6 \%$ and $91.9 \%$, respectively.

One of the most important aspects for the practicality and usefulness of diagnostic tests is the type of antigen to be used; its antigenic performance and the simplicity of its production that result in a practical method and avoid laborious and difficult procedures: ESEA antigens have all of these characteristics.

TESA antigens have been used widely for the detection of Chagas disease in different formats (Western blot, ELISA, multiple antigen binding assay: MABA) and show excellent sensitivity and specificity for all of these diagnostic assays $[16,19,35,36]$. Additionally, these antigens are used in Brazilian blood banks for confirmatory testing or to classify inconclusive results of T. cruzi infections [37]. Nevertheless, TESA production is both complicated and expensive. Thus, ESEA antigens provide an attractive alternative strategy for the diagnosis of this parasitic disease since these antigens are easier and cheaper to produce. Epimastigotes grow easily and rapidly in broth media, and high yields are obtained after the logarithmic growth phase. 
TABLE 2: Mean, standard deviation (SD), optical density range (OD), and specificity estimates for serum groups in ELISAs based on secreted and excreted antigen homogenates (ESEA antigens) from trypomastigote forms of T. cruzi.

\begin{tabular}{|c|c|c|c|c|c|c|}
\hline Group of individuals & Mean $(\mathrm{OD}) \pm \mathrm{SD}$ & $\begin{array}{l}\text { Range DO } \\
\text { min-max }\end{array}$ & $\begin{array}{c}\text { Sp \% } \\
(\mathrm{DO} 0,500)\end{array}$ & $\begin{array}{c}\text { Sens } \% \\
(\text { DO } 0,500)\end{array}$ & $\begin{array}{c}\text { Sp \% } \\
(\text { DO } 0,600)\end{array}$ & $\begin{array}{c}\text { Sens } \% \\
(\text { DO } 0,600)\end{array}$ \\
\hline $\begin{array}{l}\text { Individuals negative for T. cruzi } \\
\text { infection }(n=50)\end{array}$ & $0.339 \pm 0.222$ & $0.073-1.129$ & 80 & - & 88 & - \\
\hline $\begin{array}{l}\text { Chagas-positive individuals } \\
(n=50)\end{array}$ & $1.099 \pm 0.323$ & $0.474-1.888$ & 一 & 98 & - & 98 \\
\hline $\begin{array}{l}\text { Leishmaniasis-positive } \\
\text { individuals }(n=10)\end{array}$ & $0.617 \pm 0.244$ & $0.370-1.137$ & 30 & - & 60 & - \\
\hline $\begin{array}{l}\text { Individuals-positive for other } \\
\text { parasitic diseases }(n=10)\end{array}$ & $0.492 \pm 0.275$ & $0.205-0.921$ & 50 & - & 60 & - \\
\hline
\end{tabular}

OD: optical density; SD: standard deviation; min: minimum, max: maximum; Sens: sensitivity; Sp: specificity.

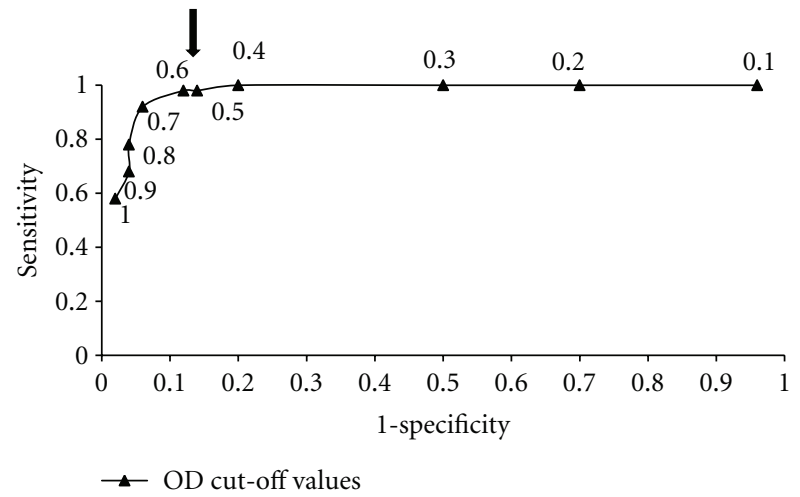

FIGURE 3: Receiver-operating characteristic curve for sensitivity and specificity (1-SP) in the enzyme-linked immunosorbent assays using ESEA antigens (AU isolate) at different arbitrary optical density (OD) cut-off values. Arrow: cutoff (0.600).

Although ESEA-ELISA showed very high sensitivity (98\%), the test had limitations as regards the specificity: six sera from healthy patients, four from patients with leishmaniasis, and four with other parasitic diseases gave false positive reactions. As has been reported previously, limitations associated with specificity in the diagnosis of Chagas disease have been reported by other authors, and patients infected with Leishmania spp. are the most likely to cross-react $[16,19,36]$. In order to correct for this error, positive ELISA-ESEA samples should be confirmed by a second serological and more specific assay such as Western blotting.

In addition to confirming the potential efficacy of the ESEA antigens for the diagnosis of T. cruzi infections, we also demonstrated their advantages. Large amounts of ESEA antigens were obtained using a simple and inexpensive protocol (six $25 \mathrm{~cm}^{2}$ flasks yielded approximately $60 \mathrm{~mL}$ of ESEA proteins at $16.18 \mu \mathrm{g} / \mathrm{mL}$ ). The yield from six flasks would permit the coating of approximately 21 ELISA plates and the performance of 851 tests.

In summary, we believe that although TESA antigens have a superior performance in terms of sensitivity and specificity, our ELISA-ESEA antigens have several advantages. These include efficiency, ease of production, and high yields that ensure sufficient assays can be undertaken. Moreover, our study is the first to describe the immunogenic bands in the ESEA antigens that are recognized by chagasic sera. A study validation of ELISA-ESEA in the field is clearly needed but these antigens certainly represent an alternative for Chagas disease diagnosis in laboratories with scarce resources.

\section{Acknowledgments}

The authors thank Dr. Herbert Tanowitz (Albert Einstein College of Medicine, NY, USA) for providing the T. cruzi strains. This work was supported by Health Canada Grant no. HT070-010033 and partially supported by a Grant from FONACIT (Misión Ciencias) no. 2007001425 and a Grant no. CI-2-040102-1417-08 from Research Commission, Núcleo de Sucre, Universidad de Oriente, Venezuela to Dr. Mariolga Berrizbeitia.

\section{References}

[1] WHO, "Chagas disease: Control and elimination," http://apps .who.int/gb/ebwha/pdf_files/WHA63/A63_17-en.pdf, 2010.

[2] F. Lopez-Antunado, H. Rangel-Flores, and C. Ramos, "Diagnosis of Chagas' Disease," Revista Latinoamericana de Microbiología, vol. 42, pp. 121-129, 2000.

[3] M. Berrizbietia, M. Ndao, M. Gottschalk et al., "Development and comparison of enzyme immunoassays for diagnosis of Chagas' disease using fixed forms of Trypanosoma cruzi (Epimastigotes, Amastigotes, and Trypomastigotes) and assessment of antigen stability for the three assays," Journal of Clinical Microbiology, vol. 42, no. 4, pp. 1766-1769, 2004.

[4] M. Schechter, J. E. Flint, and A. Voller, "Purified Trypanosoma cruzi specific glycoprotein for discriminative serological diagnosis of South American trypanosomiasis (Chagas' disease)," The Lancet, vol. 2, no. 8356, pp. 939-941, 1983.

[5] I. S. Marcipar, E. Welchen, C. Roodveldt, A. J. Marcipar, and A. M. Silber, "Purification of the $67-\mathrm{kDa}$ lectin-like glycoprotein of Trypanosoma cruzi, LLGP-67, and its evaluation as a relevant antigen for the diagnosis of human infection," FEMS Microbiology Letters, vol. 220, no. 1, pp. 149-154, 2003.

[6] J. M. Peralta, M. D. G. M. Teixeira, W. G. Shreffler et al., "Serodiagnosis of Chagas' disease by enzyme-linked immunosorbent assay using two synthetic peptides as antigens," Journal of Clinical Microbiology, vol. 32, no. 4, pp. 971-974, 1994. 
[7] W. S. F. Meira, L. M. C. Galvão, E. D. Gontijo, G. L. L. Machado-Coelho, K. A. Norris, and E. Chiari, "Use of the Trypanosoma cruzi Recombinant Complement Regulatory Protein To Evaluate Therapeutic Efficacy following Treatment of Chronic Chagasic Patients," Journal of Clinical Microbiology, vol. 42, no. 2, pp. 707-712, 2004.

[8] E. S. Umezawa, S. F. Bastos, J. R. Coura et al., "An improved serodiagnostic test for Chagas' disease employing a mixture of Trypanosoma cruzi recombinant antigens," Transfusion, vol. 43, no. 1, pp. 91-97, 2003.

[9] W. S. F. Meira, L. M. C. Galvão, E. D. Gontijo, G. L. L. Machado-Coelho, K. A. Norris, and E. Chiari, "Trypanosoma cruzi recombinant complement regulatory protein: a novel antigen for use in an enzyme-linked immunosorbent assay for diagnosis of Chagas' disease," Journal of Clinical Microbiology, vol. 40, no. 10, pp. 3735-3740, 2002.

[10] E. D. Silva, V. R. A. Pereira, J. A. S. Gomes et al., "Use of the EIE-recombinant-Chagas-biomanguinhos kit to monitor cure of human Chagas' disease," Journal of Clinical Laboratory Analysis, vol. 16, no. 3, pp. 132-136, 2002.

[11] A. W. Ferreira, Z. R. Belem, E. A. Lemos, S. G. Reed, and A. Campos-Neto, "Enzyme-linked immunosorbent assay for serological diagnosis of chagas' disease employing a Trypanosoma cruzi recombinant antigen that consists of four different peptides," Journal of Clinical Microbiology, vol. 39, no. 12, pp. 4390-4395, 2001.

[12] Y. M. Gomes, V. R. A. Pereira, M. Nakazawa et al., "Serodiagnosis of chronic Chagas infection by using EIE-RecombinantChagas-Biomanguinhos kit," Memorias do Instituto Oswaldo Cruz, vol. 96, no. 4, pp. 497-501, 2001.

[13] J. F. Da Silveira, E. S. Umezawa, and A. O. Luquetti, "Chagas disease: recombinant Trypanosoma cruzi antigens for serological diagnosis," Trends in Parasitology, vol. 17, no. 6, pp. 286-291, 2001.

[14] R. L. Houghton, D. R. Benson, L. Reynolds et al., "Multiepitope synthetic peptide and recombinant protein for the detection of antibodies to Trypanosoma cruzi in patients with treated or untreated Chagas' disease," Journal of Infectious Diseases, vol. 181, no. 1, pp. 325-330, 2000.

[15] M. Berrizbeitia, M. Ndao, J. Bubis et al., "Field evaluation of four novel enzyme immunoassays for Chagas' disease in Venezuela blood banks: comparison of assays using fixed-epimastigotes, fixed-trypomastigotes or trypomastigote excreted-secreted antigens from two Trypanosoma cruzi strains," Transfusion Medicine, vol. 16, no. 6, pp. 419-431, 2006.

[16] M. Berrizbeitia, M. Ndao, J. Bubis et al., "Purified excretedsecreted antigens from Trypanosoma cruzi trypomastigotes as tools for diagnosis of Chagas' disease," Journal of Clinical Microbiology, vol. 44, no. 2, pp. 291-296, 2006.

[17] N. Kesper Jr., K. A. De Almeida, A. M. S. Stolf, and E. S. Umezawa, "Immunoblot analysis of trypomastigote excreted-secreted antigens as a tool for the characterization of Trypanosoma cruzi strains and isolates," Journal of Parasitology, vol. 86 , no. 4 , pp. 862-867, 2000.

[18] E. S. Umezawa, M. S. Nascimento, N. Kesper Jr. et al., "Immunoblot assay using excreted-secreted antigens of Trypanosoma cruzi in serodiagnosis of congenital, acute, and chronic Chagas' disease," Journal of Clinical Microbiology, vol. 34, no. 9, pp. 2143-2147, 1996.

[19] E. S. Umezawa, M. S. Nascimento, and A. M. S. Stolf, "Enzyme-linked immunosorbent assay with Trypanosoma cruzi excreted-secreted antigens (TESA-ELISA) for serodiagnosis of acute and chronic Chagas' disease," Diagnostic
Microbiology and Infectious Disease, vol. 39, no. 3, pp. 169-176, 2001.

[20] A. M. Lissaldo, S. Hoshino-Shimizu, E. S. Umezawa, and A. M. Stolf, "Alkaline soluble Trypanosoma cruzi epimastigote antigen (ASEA) applied to Dot-ELISA.," Revista do Instituto de Medicina Tropical de Sao Paulo, vol. 36, no. 2, pp. 163-166, 1994.

[21] I. C. Almeida, D. T. Covas, L. M. T. Soussumi, and L. R. Travassos, "A highly sensitive and specific chemiluminescent enzyme-linked immunosorbent assay for diagnosis of active Trypanosoma cruzi infection," Transfusion, vol. 37, no. 8, pp. 850-857, 1997.

[22] C. D. Partel and C. L. Rossi, "A rapid, quantitative enzymelinked immunosorbent assay (ELISA) for the immunodiagnosis of Chagas' disease," Immunological Investigations, vol. 27, no. 1-2, pp. 89-96, 1998.

[23] R. T. Pinho, R. C. Pedrosa, P. Costa-Martins, and L. R. R. Castello-Branco, "Saliva ELISA: a method for the diagnosis of chronic Chagas disease in endemic areas," Acta Tropica, vol. 72, no. 1, pp. 31-38, 1999.

[24] L. H. Tobler, P. Contestable, L. Pitina et al., "Evaluation of a new enzyme-linked immunosorbent assay for detection of Chagas antibody in US blood donors," Transfusion, vol. 47, no. 1, pp. 90-96, 2007.

[25] N. Gonzalez, I. Galindo, P. Guevara et al., "Identification and detection of Trypanosoma cruzi by using a DNA amplification fingerprint obtained from the ribosomal intergenic spacer," Journal of Clinical Microbiology, vol. 32, no. 1, pp. 153-158, 1994.

[26] R. P. Souto and B. Zingales, "Sensitive detection and strain classification of Trypanosoma cruzi by amplification of a ribosomal RNA sequence," Molecular and Biochemical Parasitology, vol. 62, no. 1, pp. 45-52, 1993.

[27] U. K. Laemmli, "Cleavage of structural proteins during the assembly of the head of bacteriophage T4," Nature, vol. 227, no. 5259, pp. 680-685, 1970.

[28] H. Towbin, T. Staehelin, and J. Gordon, "Electrophoretic transfer of proteins from polyacrylamide gels to nitrocellulose sheets: procedure and some applications," Proceedings of the National Academy of Sciences of the United States of America, vol. 76, no. 9, pp. 4350-4354, 1979.

[29] J. Crowther, The ELISA Guidebook, Springer, New York, NY, USA, 1st edition, 2000.

[30] Y. Campos, L. Briceño, K. Reina, K. Figarella, J. L. Pérez, and W. Mosca, "Serological diagnosis of Chagas disease: evaluation and characterisation of a low cost antigen with high sensitivity and specificity," Memorias do Instituto Oswaldo Cruz, vol. 104, no. 6, pp. 914-917, 2009.

[31] W. R. Cuna, C. Rodriguez, F. Torrico, D. Afchain, M. Loyens, and P. Desjeux, "Evaluation of a competitive antibody enzyme immunoassay for specific diagnosis of Chagas' disease," Journal of Parasitology, vol. 75, no. 3, pp. 357-359, 1989.

[32] D. Cannaova, C. Aguilar, M. Pacheco, M. Simons, and M. Medina, "Validación del Inmuno Ensayo Enzimático (ELISA) y Hemoaglutinación Indirecta (HAI) para el Serodiagnóstico de la Enfermedad de Chagas," Salus, no. 3, pp. 4-9, 2002.

[33] S. Telles, T. Abate, T. Slezynger, and D. A. Henriquez, "Trypanosoma cruzi ubiquitin as an antigen in the differential diagnosis of Chagas disease and leishmaniasis," FEMS Immunology and Medical Microbiology, vol. 37, no. 1, pp. 23$28,2003$.

[34] M. Schechter, A. O. Luquetti, J. M. Rezende, A. Rassi, and M. A. Miles, "Further evaluation of lectin affinity purified glycoprotein (GP90) in the enzyme linked immunosorbent 
assay (ELISA) for diagnosis of Trypanosoma cruzi infection," Transactions of the Royal Society of Tropical Medicine and Hygiene, vol. 79, no. 5, pp. 637-640, 1985.

[35] M. Berrizbeitia, B. J. Ward, J. Bubis et al., " $85-\mathrm{kDa}$ protein of Trypanosoma cruzi purified by affinity chromatography used in the multiple antigen binding assay (MABA) for the diagnosis of T. cruzi infection in a Venezuelan rural community," Parasitology Research, vol. 106, no. 5, pp. 11271134, 2010.

[36] M. Nakazawa, D. S. Rosa, V. R. A. Pereira et al., "Excretorysecretory antigens of Trypanosoma cruzi are potentially useful for serodiagnosis of chronic Chagas' disease," Clinical and Diagnostic Laboratory Immunology, vol. 8, no. 5, pp. 10241027, 2001.

[37] E. P. Silveira-Lacerda, A. G. Silva, S. F. Junior et al., "Chagas' disease: application of TESA-blot in inconclusive sera from a Brazilian blood bank," Vox Sanguinis, vol. 87, no. 3, pp. 204207, 2004 


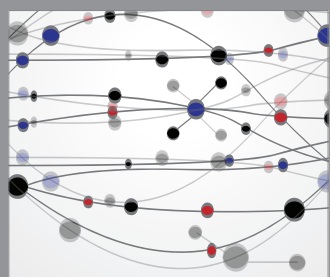

The Scientific World Journal
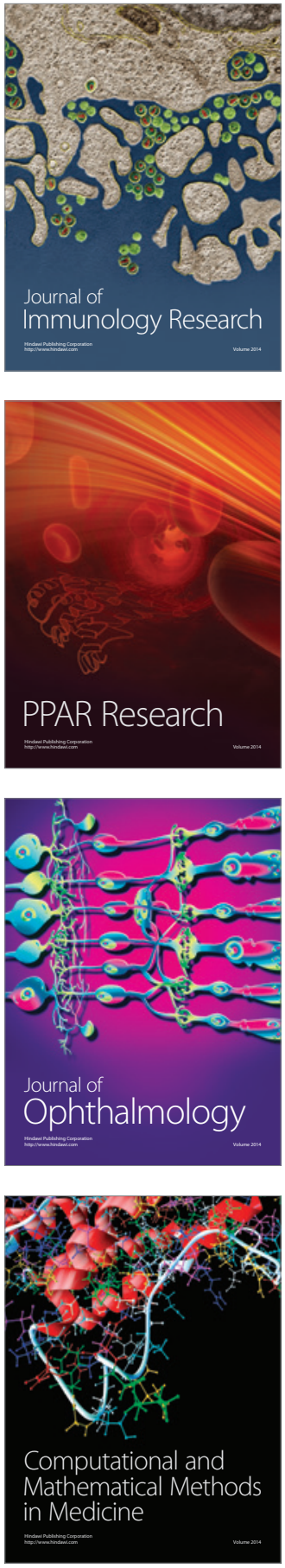

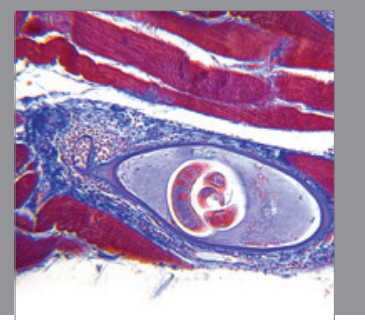

Gastroenterology

Research and Practice
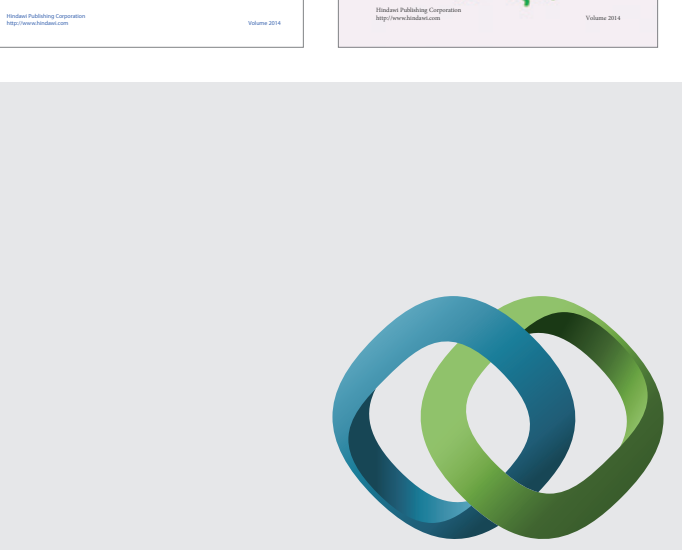

\section{Hindawi}

Submit your manuscripts at

http://www.hindawi.com
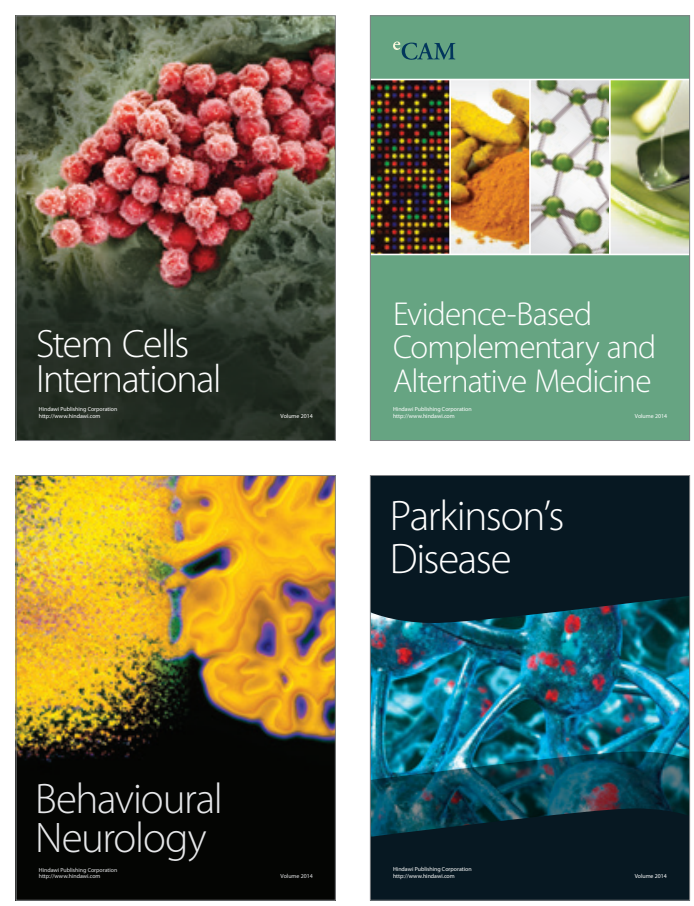

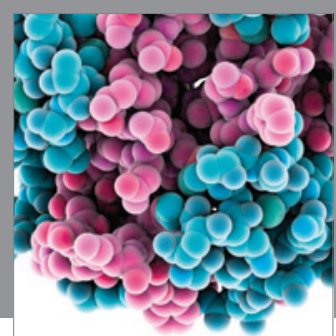

Journal of
Diabetes Research

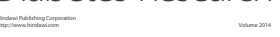

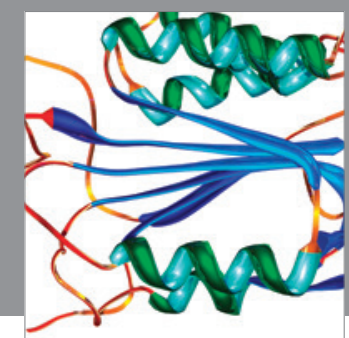

Disease Markers
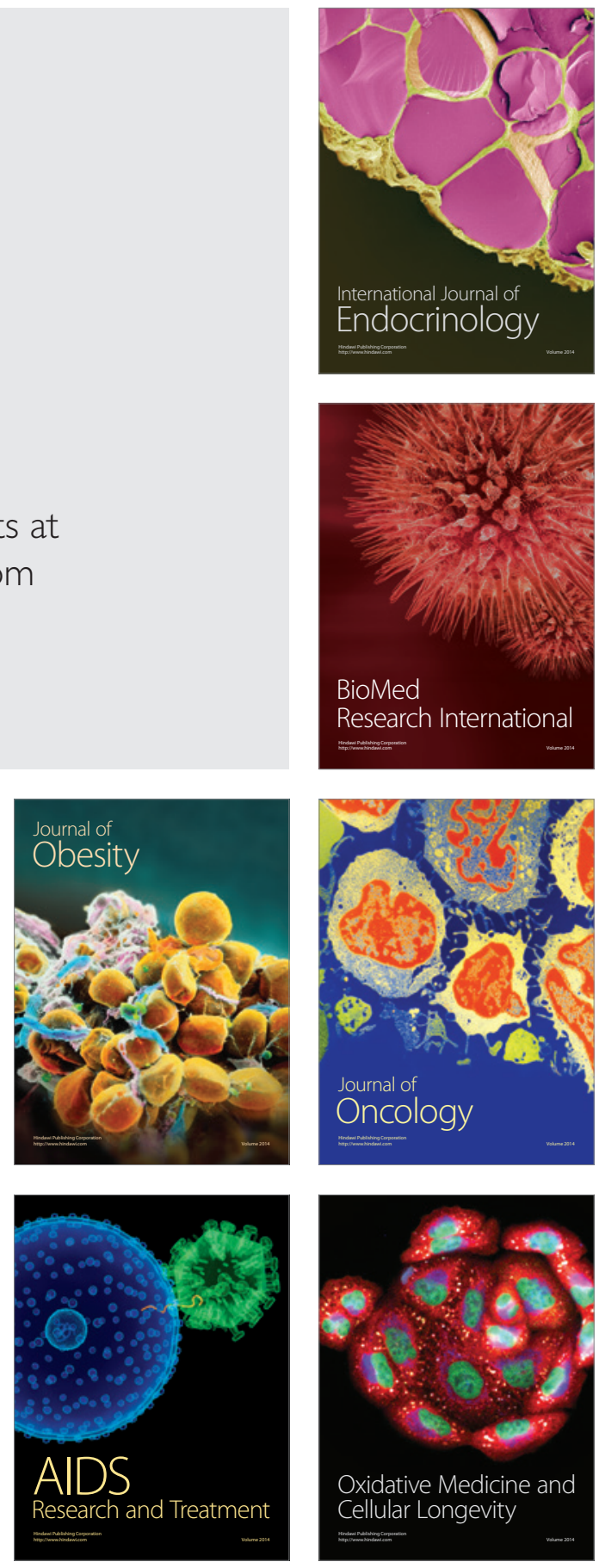